\title{
Assessment of the quality of chest compressions in healthcare professionals
}

Artem Kuzovlev 1,2, Viktor Moroz 1,2, Arkady Goloubev ${ }^{1,2}$, Sergey Abdusalamov², Kirill Kuzmichev ${ }^{2}$, Evgeny Tishkov ${ }^{3}$

Federal Research and Clinical Center of Intensive Care Medicine and Rehabilitology, Moscow, Russia 2 Russian National Ressucitation Council, Moscow, Russia

${ }^{3}$ A.I. Evdokimov Moscow medical dental university, Moscow, Russia

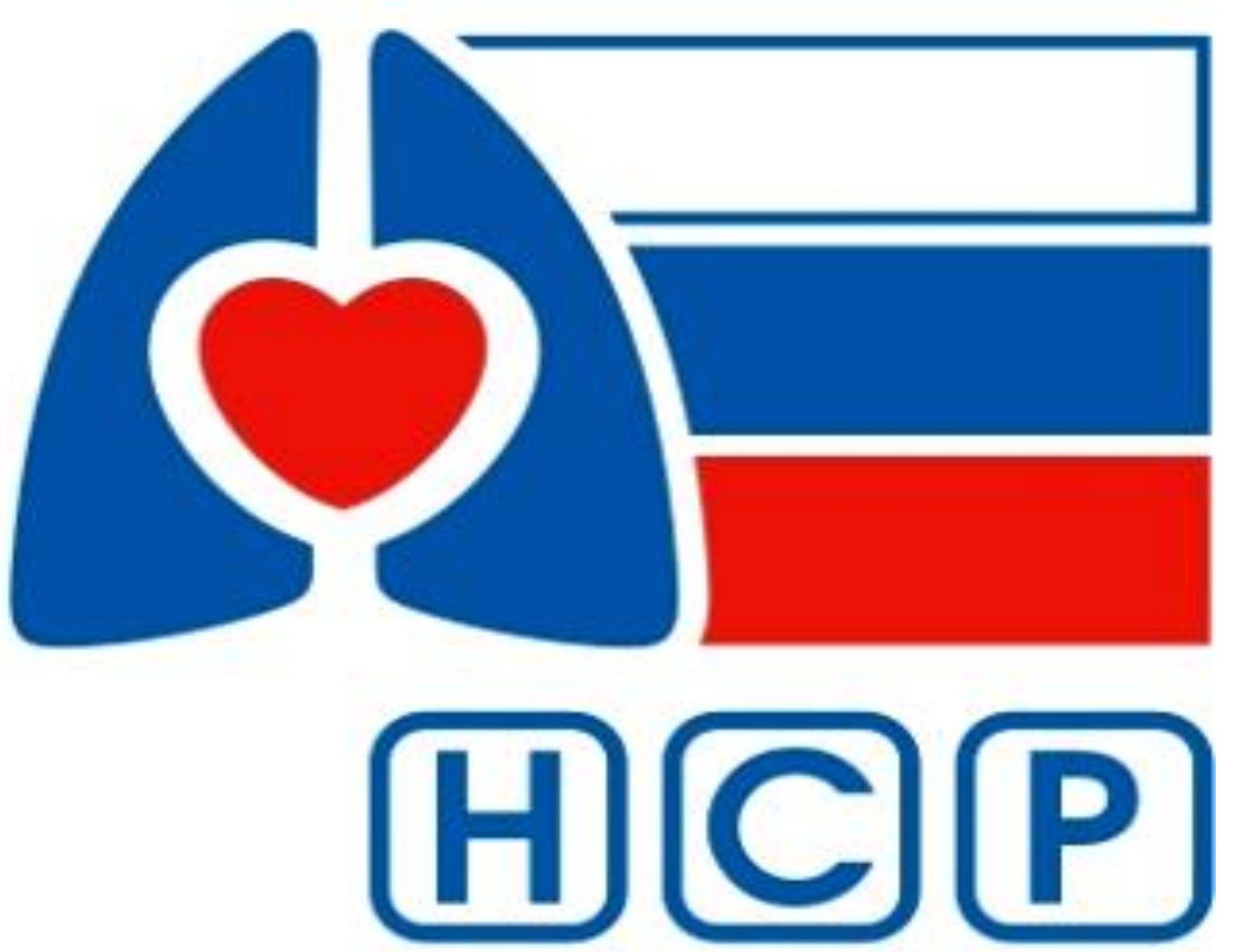

НАЦИОНААЬНЫЙСОВЕТ ПО РЕАНИМАЦИИ

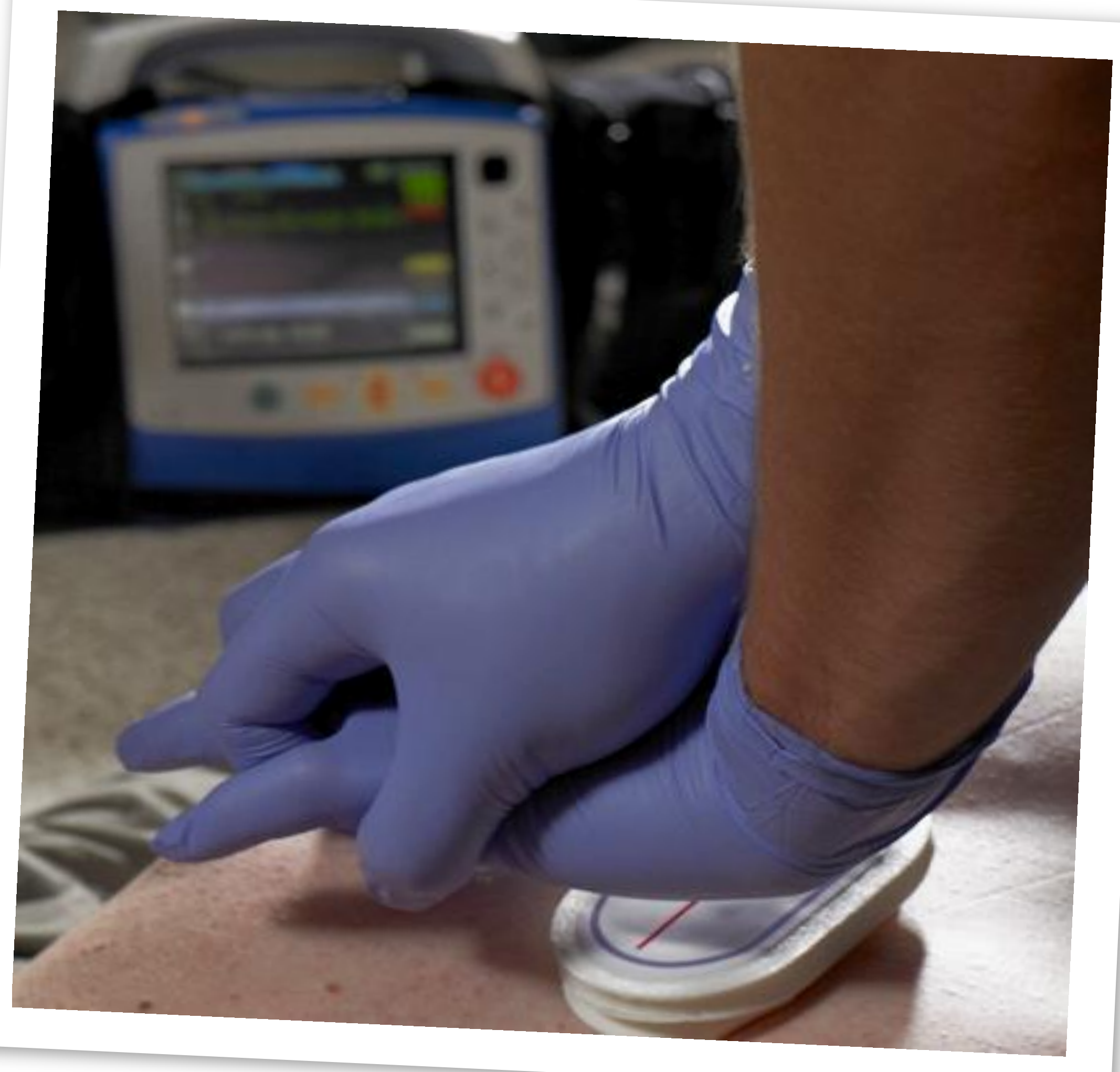

- Immediate and high-quality chest compressions (CC) make the background of in-hospital cardiopulmonary resuscitation.

- None of the studies on feedback or prompt devices has demonstrated improved survival to discharge with feedback [ERC guidelines 2015].

- The use of CPR feedback or prompt devices during CPR should only be considered as part of a broader system of care that should include comprehensive CPR quality improvement initiatives, rather than as an isolated intervention [ERC guidelines 2015].

The aim of the study was to assess the quality of chest compressions in healthcare providers in the multidisciplinary hospital.

- The study was performed in a multifield hospital in Moscow, in 2016-2017.

- 42 healthcare professional were enrolled.

- Chest compressions were assessed in 2 steps by means of CPR-challenge tool: 2 mins of CC without feedback then 2 mins of CC with feedback on rate, depth and recoil

- The ERC Guidelines for Resuscitation 2015 were used as reference criteria for CC quality.

- The analysis was performed using the ZOLL RescueNet Code ReviewR software.

- Statistical analysis was performed using the Statistica 7.0 software (Mann-Whitney $U$-test). The data were presented as a mean, median $\pm 25-75$ percentiles (25-75 IQR), minimum and maximum values. The difference was considered significant at $P<0.05$.
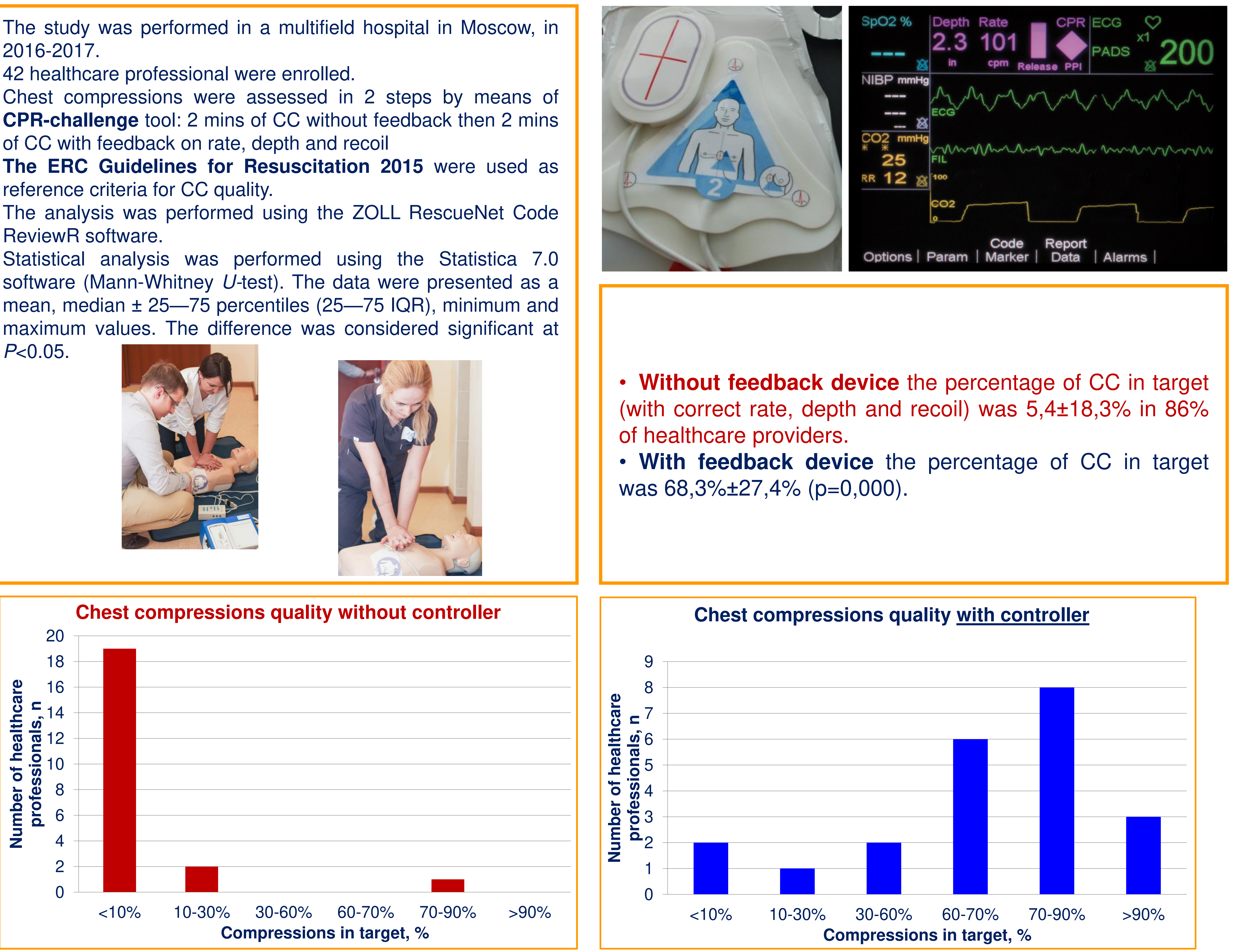

- Without feedback device the percentage of CC in target (with correct rate, depth and recoil) was $5,4 \pm 18,3 \%$ in $86 \%$ of healthcare providers.

- With feedback device the percentage of CC in target was $68,3 \% \pm 27,4 \%(p=0,000)$.

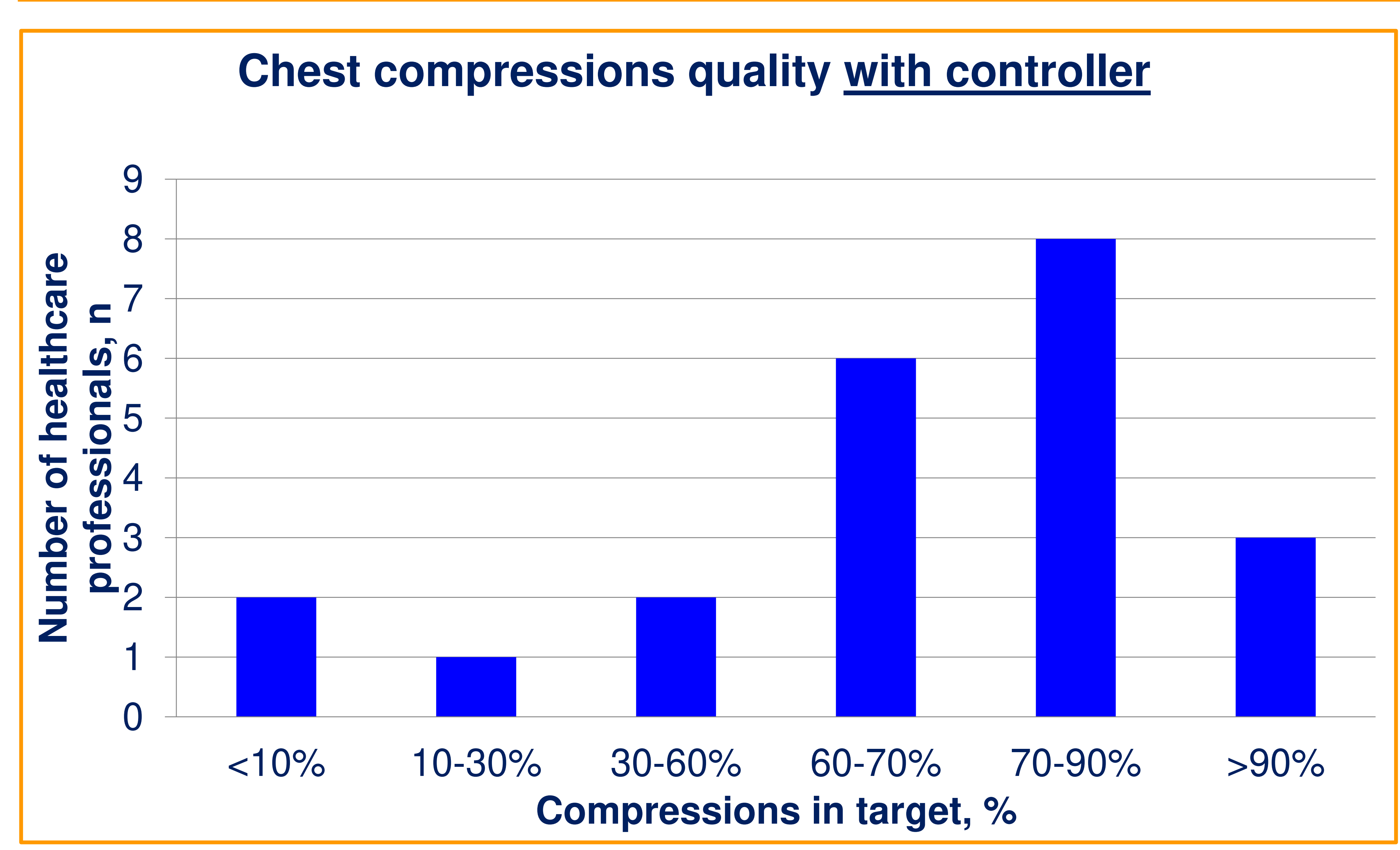

Healthcare providers have low background CC skills which improve when feedback devices are used.

Regular simulation training and retraining is required. 